\title{
Evaluación de la capacidad antioxidante y compuestos bioactivos de tumbo (Passiflora mollissima) y cerezo (Prunus serotina)
}

Juan Lopa* 1,a; María Valderrama 2,b; Nelva León 2,c; Luz Lazo ${ }^{3, \mathrm{~d}}$; Jean Pierre Llerena 1,e; Carlos Ballón 3,f; Emilio Guija-Poma 4,g

\section{RESUMEN}

Objetivo: Determinar el contenido de compuestos bioactivos y la capacidad antioxidante de los frutos tumbo (Passiflora mollissima) y cerezo (Prunus serotina).

Materiales y métodos: Estudio analítico, experimental, longitudinal y prospectivo. Los frutos cerezo y tumbo se recolectaron en las regiones de Cusco, Moquegua y Arequipa. La técnica de Folin-Ciocalteu fue empleada para determinar el contenido de fenoles, y el cloruro de aluminio se utilizó para calcular los flavonoides. La actividad antioxidante se evaluó mediante las técnicas Ferric Reducing Ability of Plasma (FRAP), 2,2-difenil-picril-hidrazil (DPPH) y sustancias reactivas al ácido tiobarbitúrico (TBARS). Para estudiar el efecto hepatoprotector de las frutas, utilizamos ratas albinas que se clasificaron en un grupo control negativo, un grupo control positivo y cuatro grupos experimentales.

Resultados: La mayor concentración de fenoles totales y flavonoides se encontró en el tumbo de la región Cusco (Quechua) (fenoles totales: 584,94 \pm 134,62 mg EAG / 100 g y flavonoides :445,62 \pm 7,94 mg EQ / 100 g). Para el radical DPPH, el valor IC50 del tumbo de la región Arequipa (Yunga) fue 0,41 \pm 0,01 mg / mL. El tumbo de la región Cusco (Quechua) mostró el valor FRAP más alto $(8,38 \pm 0,32 \mathrm{mmol} \mathrm{Fe}+/ 100 \mathrm{~g})$. El cerezo de la región de Arequipa (Yunga) presentó la mayor concentración de fenoles totales $(181,81 \pm 34,1 \mathrm{mg}$ EAG / $100 \mathrm{~g})$ y flavonoides $(205,18 \pm 77,8 \mathrm{mg}$ EQ / $100 \mathrm{~g})$. El cerezo de Arequipa (Yunga) mostró una actividad antioxidante significativa al DPPH $(2,1 \pm 0,01 \mathrm{mg} / \mathrm{mL})$, mientras que la capacidad antioxidante del cerezo de la región Cusco (Quechua), evaluada con la técnica FRAP, alcanzó un valor de 1,59 0,2 mmol Fe ${ }^{2+} / 100$ g. Las diferencias observadas fueron estadísticamente significativas. El tumbo mostró un mejor efecto hepatoprotector que el cerezo.

Conclusiones: El tumbo de la región Cusco (Quechua) es una fuente importante de compuestos antioxidantes y muestra una elevada capacidad antioxidante (FRAP), mientras que el cerezo de la región Arequipa (Yunga) tiene un alto contenido de compuestos antioxidantes y una mayor capacidad antioxidante (DPPH).

Palabras clave: Antioxidantes; Fenoles; Flavonoides; Prunus serotina (Fuente: DeCS BIREME).

\section{Evaluation of the antioxidant capacity and bioactive compounds of banana passionfruit (Passiflora mollissima) and black cherry (Prunus serotina)}

\section{ABSTRACT}

Objective: To determine the content of bioactive compounds and antioxidant capacity of the banana passionfruit (Passiflora mollissima) and black cherry (Prunus serotina).

Materials and methods: An analytical, experimental, longitudinal and prospective study. The black cherries and banana passionfruits were collected in the Cusco, Moquegua and Arequipa regions. The content of phenols and flavonoids were determined using the Folin-Ciocalteu reagent and aluminum chloride method, respectively. The antioxidant activity was evaluated using the ferric reducing ability of plasma (FRAP), 2,2-diphenyl-1-picryl-hydrazyl (DPPH) and thiobarbituric acid

\footnotetext{
1 Universidad Nacional de San Agustín, Facultad de Ciencias Naturales y Formales, Departamento Académico de Química. Arequipa, Perú. 2 Universidad Nacional de San Agustín. Facultad de Ciencias Biológicas, Departamento Académico de Biología. Arequipa, Perú.

3 Universidad Nacional de San Agustín, Facultad de Ciencias Biológicas, Departamento Académico de Ciencias de la Nutrición. Arequipa, Perú. 4 Universidad de San Martín de Porres, Facultad de Medicina Humana, Centro de Investigación de Bioquímica y Nutrición. Lima, Perú. a Licenciado Químico, Doctor en Ciencias y Tecnologías Medioambientales.

b Bióloga, Doctora en Ciencias Biológicas con mención en Ingeniería Genética. c Licenciada en Nutrición y Dietética, Doctora en Ciencias: Salud Pública. d Licenciada en Nutrición Humana, Magister en Bioquímica y Biología Molecular. e Estudiante de la Escuela Profesional de Química, Asistente de Investigación.

f Estudiante de la Escuela Profesional de Ciencias de la Nutrición, Asistente de Investigación. g Químico Farmacéutico, Doctor en Farmacia y Bioquímica.

* Autor corresponsal.
} 
reactive substance (TBARS) techniques. Albino rats classified into a negative control group, a positive control group and four experimental groups were used to study the hepatoprotective effect of the fruits.

Results: Banana passionfruits from the Cusco region (Quechua) showed the highest concentration of total phenols (584.94 $\pm 134.62 \mathrm{mg} \mathrm{GAE} / 100 \mathrm{~g})$ and flavonoids (445.62 $\pm 7.94 \mathrm{mg} \mathrm{QE} / 100 \mathrm{~g})$. Concerning the DPPH radical, the IC value of banana passionfruits from the Arequipa region (Yunga) was found to be $0.41 \pm 0.01 \mathrm{mg} / \mathrm{mL}$. Banana passionfruits from the Cusco region (Quechua) showed the highest FRAP value $(8.38 \pm 0.32 \mathrm{mmol} \mathrm{Fe}+/ 100 \mathrm{~g})$. Black cherries from the Arequipa region (Yunga) had the highest concentration of total phenols $(181.81 \pm 34.1 \mathrm{mg} \mathrm{GAE} / 100 \mathrm{~g})$ and flavonoids $(205.18 \pm 77.8 \mathrm{mg} \mathrm{QE} / 100 \mathrm{~g})$. They also showed a significant antioxidant activity regarding the DPPH $(2.1 \pm 0.01 \mathrm{mg} / \mathrm{mL})$, while the antioxidant capacity of black cherries from the Cusco region (Quechua), which was evaluated with the FRAP method, achieved a value of $1.59 \pm 0.2 \mathrm{mmol} \mathrm{Fe}{ }^{2}+100 \mathrm{~g}$. The observed differences were statistically significant. Banana passionfruits showed a better hepatoprotective effect than black cherries.

Conclusions: Banana passionfruits from the Cusco region (Quechua) are an important source of antioxidant compounds and show a high antioxidant capacity (FRAP), while black cherries from the Arequipa region (Yunga) have a high content of antioxidant compounds and a higher antioxidant capacity (DPPH).

Keywords: Antioxidants; Phenols; Flavonoids; Prunus serotina (Source: MeSH NLM).

\section{INTRODUCCIÓN}

Diversas enfermedades están estrechamente relacionadas con la generación de especies reactivas de oxígeno (ROS, del inglés reactive oxygen species). Estos compuestos se caracterizan por tener un electrón desapareado en su orbital externo, condición que los torna altamente reactivos ${ }^{(1,2)}$. En el ser humano los ROS se generan en la cadena respiratoria mitocondrial, la reacción del peróxido de hidrógeno con iones ferrosos, la interacción de ascorbato con iones cúpricos ${ }^{(3)}$, etc. Los ROS ejercen acción oxidante sobre las proteínas, lípidos y ácidos nucleicos ${ }^{(4)}$. Además, afectan diversas vías de señalización celular en las que intervienen el factor nuclear kappa B (NF- $\mathcal{B} B$ ), el factor 2 relacionado a NF-E2 (Nrf2), la quinasa Jun $\mathrm{N}$-terminal (JNK) ${ }^{(5)}$, entre otros; y ocasiona así el estrés oxidativo, situación en la que no se dispone de una adecuada defensa antioxidante ${ }^{(6)}$. Esta defensa está constituida por enzimas como la superóxido dismutasa, catalasa glutatión reductasa, etc., así como por compuestos no enzimáticos como el ácido úrico, transferrina, ceruloplasmina, glutatión, etc.

Los humanos no cuentan con una defensa antioxidante eficiente para evitar el daño ocasionado por los ROS. Por ello, deben ingerir sustancias que les proporcionen una adecuada protección antioxidante. Las frutas y verduras son fuentes importantes de sustancias antioxidantes, ya que sus metabolitos secundarios son flavonoides, polifenoles, taninos, vitamina $\mathrm{C}$, licopenos, antocianinas, etc., sustancias que, entre otras funciones, neutralizan el efecto dañino de los ROS en el ser humano ${ }^{(7-9)}$.

Los ROS pueden alterar las proteínas, propiciar la lipoperoxidación y dañar el ADN. Por lo tanto, están vinculados a una gran diversidad de enfermedades del ser humano ${ }^{(10,11)}$, tales como cáncer, diabetes mellitus, psoriasis, aterosclerosis, obesidad, cataratas, etc. Asimismo, los estudios epidemiológicos muestran que las personas que ingieren alimentos como frutas y verduras tienen menos posibilidades de padecer enfermedades crónicas no transmisibles ${ }^{(12)}$.

Diversas publicaciones han descrito las propiedades antioxidantes del tumbo ${ }^{(13)}$, lo que ha permitido conocer la considerable variación en la composición química y las propiedades antioxidantes de esta fruta, que están relacionadas al contenido de nutrientes en el terreno, diferencias geográficas e influencia de la luz solar. Respecto al cerezo, se ha observado una gran diversidad genética en todo el mundo ${ }^{(14)}$ que se investiga para establecer si esta diferencia se expresa en la morfología del fruto, lo que significaría que existen procesos adaptativos a agroecosistemas diferentes. En relación con los compuestos antioxidantes (fenoles totales, flavonoides) y con la capacidad antioxidante ${ }^{(15)}$, se ha observado que existen considerables diferencias vinculadas a la naturaleza del terreno, la altitud y la variabilidad genética, entre otros factores.

En las diferentes regiones del Perú, durante todo el año, crece una gran variedad de frutos. En algunos casos, para su desarrollo no se emplean fertilizantes ni plaguicidas, como ocurre con el aguaymanto, la tuna o el camu camu. El tumbo y el cerezo (también llamado capulí o guinda, según la zona de producción) son los frutos estudiados en esta investigación y cuyas propiedades antioxidantes se desconocen, especialmente si consideramos que se han recolectado en distintos pisos ecológicos. En tal sentido, se 
ha evaluado el contenido de antioxidantes de ambos frutos, su capacidad antioxidante y el efecto que muestran en ratas sometidas a la acción hepatotóxica del paracetamol.

\section{MATERIALES Y MÉTODOS}

\section{Diseño y población de estudio}

El estudio es de tipo analítico, transversal y prospectivo experimental. La identificación taxonómica de los frutos Passiflora mollissima (tumbo) y Prunus serotina (cerezo) fue realizada en el Herbarium Areqvipense (HUSA) de la Universidad Nacional de San Agustín de Arequipa. Los frutos se colectaron en los valles interandinos de las regiones Arequipa, Cusco y Moquegua. Las ubicaciones geográficas fueron las siguientes: Arequipa (Yunga) $16^{\circ} 24^{\prime} 40.26 " \mathrm{~S}$; $71^{\circ} 38^{\prime} 42.96^{\prime \prime}$ O, Arequipa (Quechua) $15^{\circ} 6^{\prime} 37.296^{\prime \prime} \mathrm{S} ; 72^{\circ}$ $31^{\prime} 3.507^{\prime \prime}$ O, Arequipa (Suni) $15^{\circ} 14^{\prime} 9.22^{\prime \prime} \mathrm{S} ; 73^{\circ} 4^{\prime} 21.154^{\prime \prime}$ O, Cusco (Quechua) $14^{\circ} 23^{\prime} 26.185^{\prime \prime} \mathrm{S} ; 2^{\circ} 2^{\circ} 22.938^{\prime \prime} \mathrm{O}$, Cusco (Suni) $14^{\circ} 17^{\prime} 1^{\prime \prime} \mathrm{S} ; 72^{\circ} 02^{\prime} 25^{\prime \prime}$ O, Moquegua (Quechua) $16^{\circ} 39^{\prime} 21.78^{\prime \prime} \mathrm{S} ; 70^{\circ} 57^{\prime} 21.84^{\prime \prime} 0$ y Moquegua (Suni) $16^{\circ} 35^{\circ}$ $46.44^{\prime \prime} \mathrm{S} ; 71^{\circ} 13^{\prime} 28.86^{\prime \prime} \mathrm{O}$.

La metodología descrita por Carrión y García (17) fue utilizada para preparar los extractos. Pesamos $2,5 \mathrm{~g}$ de pulpa de los frutos que luego fueron macerados con una solución hidroalcohólica. Se mantuvieron en oscuridad y refrigeración por 24 horas y se centrifugaron a 5000 r. p. m. durante 20 minutos. Los sobrenadantes se guardaron a $0{ }^{\circ} \mathrm{C}$. Este procedimiento se repitió por tres veces.

Se utilizaron 36 ratas albinas adultas machos de la cepa Wistar de tres meses de edad con pesos entre 250 y 380 gramos, alojadas en jaulas mantenidas en condiciones de laboratorio estandarizadas (ciclos de 12 horas de luz/oscuridad, temperatura $22 \pm 2{ }^{\circ} \mathrm{C}$ y humedad $51 \%$ ), con acceso a agua ad libitum y alimento estándar para ratas obtenido del bioterio de la Universidad Católica de Santa María, donde se realizó el estudio. Los animales estuvieron en un período de adaptación previo de siete días.

Los tratamientos fueron administrados por vía oral durante siete días. Al grupo control negativo se le administró 1 $\mathrm{mL}$ de agua destilada. El grupo control positivo recibió paracetamol en una dosis de $200 \mathrm{mg} / \mathrm{kg}$ de peso corporal. A todos los grupos experimentales se les administró paracetamol $(200 \mathrm{mg} / \mathrm{kg}$ de peso corporal) y además, $200 \mathrm{mg} / \mathrm{kg}$ de peso corporal de tumbo liofilizado (grupo experimental I), $400 \mathrm{mg} / \mathrm{kg}$ de peso corporal de tumbo liofilizado (grupo experimental II), $300 \mathrm{mg} / \mathrm{kg}$ de peso corporal de cerezo liofilizado (grupo experimental III) y $600 \mathrm{mg} / \mathrm{kg}$ de peso corporal de cerezo liofilizado (grupo experimental IV). Concluido el tratamiento, las ratas, previamente anestesiadas con cloroformo, fueron sacrificadas, y reservamos una muestra de sangre y una parte del hígado.

\section{VARIABLES Y MEDICIONES}

\section{Reactivos químicos}

El etanol era grado HPLC y el metanol, grado HPLC; el carbonato de sodio y el sulfato ferroso se adquirieron de JT Baker. El paracetamol, quercetina, nitrito de sodio, cloruro de aluminio, 2,2-difenil-picril-hidrazil (DPPH), 2,4,6-tripiridil-s-triazina-Fe ${ }^{3+} \quad$ (TPTZ) y 1,1,3,3-tetraetoxipropano eran de la marca Sigma Aldrich. El ácido gálico fue de Fluka Analytica. El hidróxido de sodio fue de Macron Fine Chemicals. El ácido acético glacial fue de Fisher Alert. El n-butanol era un producto de Scharlau. El Folin-Ciocalteu, cloruro férrico, ácido tiobarbitúrico y butil hidroxitolueno se adquirieron en Milipore Corporation. Todos los reactivos fueron de grado para análisis.

Los extractos hidroalcohólicos de ambos frutos se utilizaron para realizar las determinaciones analíticas de fenoles totales y flavonoides, así como la capacidad antioxidante mediante las técnicas DPPH, FRAP y TBARS.

\section{Determinación de fenoles totales}

Los fenoles totales se calcularon con el método descrito por Singleton y Rossi ${ }^{(18)}$. Para ello, se midió $1 \mathrm{~mL}$ del reactivo Folin-Ciocalteu al $10 \%$, luego $100 \mu \mathrm{L}$ de la muestra, y agregamos $1 \mathrm{~mL}$ de la solución de carbonato de sodio $7,5 \%, 2,9 \mathrm{~mL}$ de agua supra pura hasta completar el volumen, luego se colocó en baño maría a $45{ }^{\circ} \mathrm{C} \times 15$ minutos y se leyó en el espectrofotómetro (Shimadzu UV 1800) a una longitud de onda a $725 \mathrm{~nm}$. Elaboramos una curva de calibración con una solución patrón recién preparada de ácido gálico (250 ppm; 0,25 mg/mL); para este propósito, se utilizaron concentraciones comprendidas entre 50 y 300 ppm. Los resultados se expresan como mg equivalentes de ácido gálico/100 g de muestra fresca (mg EAG/100g).

\section{Determinación de flavonoides}

Los flavonoides se calcularon con el método descrito por Zhishen ${ }^{(19)}$. Se elaboró una curva patrón con quercetina, luego se agregó $1,250 \mathrm{~mL}$ de agua supra pura y nitrito de sodio al $5 \%$ a $0,250 \mathrm{~mL}$ de muestra, y todo ello quedó en reposo por 5 minutos. Se añadió cloruro de aluminio al $10 \%$ y se dejó en reposo por 6 minutos. Por último, se agregó $0,5 \mathrm{~mL}$ de hidróxido de sodio $1 \mathrm{~N}$ y $0,300 \mathrm{~mL}$ de agua destilada. La absorbancia se leyó en el espectrofotómetro a una longitud de onda de $510 \mathrm{~nm}$. Los resultados se expresan como $\mathrm{mg}$ de quercetina/100 $\mathrm{g}$ de fruta fresca (mg EQ/100g).

\section{Determinación de la capacidad antioxidante: técnica DPPH}

Para este procedimiento se empleó el método de Brand-Williams (20), que se fundamenta en la reducción del radical libre 2,2-difenil-picril-hidrazil (DPPH). Utilizamos volúmenes de muestra entre 0,02 y $0,200 \mathrm{~mL}$, 
luego se añadió $1,0 \mathrm{~mL}$ de la solución del DPPH (con D.O. aprox. 2,0 ) y metanol hasta completar un volumen final de 2,0 mL y se mantuvo en reposo en la oscuridad por 30 minutos. La absorbancia se leyó en el espectrofotómetro (longitud de onda de $515 \mathrm{~nm}$ ). Las determinaciones se realizaron por triplicado y los resultados se expresan como valor IC50, que corresponde a la concentración de la muestra problema que disminuye la densidad óptica del control en un $50 \%$.

\section{Determinación de la capacidad antioxidante: técnica FRAP}

Para este análisis se utilizó la técnica FRAP (Ferric Reducing Ability of Plasma) propuesta por Benzie y Strain (21), que emplea un tubo de ensayo con $1,0 \mathrm{~mL}$ del reactivo 2,4,6-tripiridil-s-triazina- $\mathrm{Fe}^{3+}$ (TPTZ), luego, entre 0,1 y 0,3 $\mathrm{mL}$ de muestra y agua hasta completar $2 \mathrm{~mL}$. Se colocó en baño maría a $37^{\circ} \mathrm{C}$ por 15 minutos para leer la absorbancia a $593 \mathrm{~nm}$. Con una solución de TPTZ con concentraciones distintas de sulfato ferroso se preparó una curva estándar. Los resultados se expresan como mmoles de $\mathrm{Fe}^{2+} / 100 \mathrm{~g}$ de muestra fresca.

\section{Determinación de sustancias reactivas al ácido tiobarbitúrico (TBARS)}

Empleamos el método de Ohkawa (22) modificado, que consiste en colocar en un tubo de ensayo suero $(0,5 \mathrm{~mL})$, ácido clorhídrico al $25 \%(0,5 \mathrm{~mL})$, solución de BHT al $2 \%$ $(0,045 \mathrm{~mL})$ y $500 \mu \mathrm{L}$ de TBA $(1 \% \mathrm{p} / \mathrm{v}$ en hidróxido de sodio $0,05 \mathrm{M})$. Se preparó un control con agua destilada, los tubos fueron agitados en un vortex y se colocaron en baño maría a ebullición por 10 minutos. Los tubos se enfriaron con hielo y añadimos $1,5 \mathrm{~mL}$ de $\mathrm{n}$-butanol, se agitaron nuevamente, luego se centrifugaron por 10 minutos a $4000 \mathrm{rpm}$ y se dejaron en reposo durante 30 minutos. Se separó la fase superior y se leyó a $532 \mathrm{~nm}$, las lecturas fueron comparadas con una curva patrón utilizando TEP (1,1,3,3-tetratohipoxipropano), consideramos que $1 \mathrm{~mol}$ de TEP forma, por hidrólisis, 1 mol de MDA.

\section{Estudio histopatológico}

Pequeñas porciones de los hígados de las ratas se colocaron en una solución de formol al $10 \%$, en amortiguador fosfato $0,1 \mathrm{M} \mathrm{y} \mathrm{pH} \mathrm{7,4.} \mathrm{Luego,} \mathrm{los} \mathrm{cortes} \mathrm{histológicos} \mathrm{fueron}$ coloreados con tinción de hematoxilina y eosina.

\section{Análisis estadístico}

Las determinaciones se realizaron por triplicado y se expresan como promedios \pm desviación estándar, las diferencias estadísticas se establecieron mediante ANOVA, prueba de Tukey $(p<0,05)$ y t de Student. Se utilizó un $\alpha: 0,05$.

\section{Consideraciones éticas}

El estudio fue aprobado por el Vicerrectorado de Investigación de la Universidad Nacional de San Agustín de Arequipa, y se acataron las normas internacionales sobre el cuidado y uso de animales de experimentación.

\section{RESULTADOS}

En la tabla 1 se observa el resultado del contenido de compuestos fenólicos del tumbo de Cusco (Suni) (287) y Cusco (Quechua)(584) con una diferencia estadísticamente significativa. Los valores encontrados en las muestras de las regiones Arequipa (Quechua) y Arequipa (Suni) no difieren de las de la región Moquegua (Quechua). El contenido de flavonoides hallados en los frutos provenientes de Cusco (Quechua), Moquegua (Suni), Arequipa (Yunga) y Arequipa (Suni) no fue estadísticamente significativo. Los valores más bajos de flavonoides se encontraron en las muestras originarias de las regiones Arequipa (Quechua) $(223,54 \mathrm{mg}$ $\mathrm{EQ} / 100 \mathrm{~g}$ ) y Moquegua (Quechua) $(314,72 \mathrm{mg} \mathrm{EQ} / 100 \mathrm{~g})$.

La capacidad antioxidante del tumbo se evaluó con la técnica DPPH, que se expresa como valores IC50 (mg/mL). Para realizar las comparaciones se utilizó un análisis de varianza complementado con el test de Tukey, que evidenció que la muestra de la región Cusco (Suni) tiene el mayor valor IC50, seguido de Arequipa (Quechua) y las otras regiones, como Arequipa (Suni), Cusco (Quechua) y Moquegua (Quechua), con promedios menores; las muestras de Arequipa (Yunga) y Moquegua (Suni) son las que reportaron los valores menores (IC50: 0,41 y $0,45 \mathrm{mg} / \mathrm{mL}$, respectivamente), es decir, que corresponden a las muestras con mayor capacidad antioxidante (Tabla 1).

Al evaluar la capacidad antioxidante del tumbo con la técnica FRAP, observamos que la muestra de la región Cusco (Quechua) tenía la mayor capacidad antioxidante (8,32 mmoles de $\mathrm{Fe}^{2+} / 100 \mathrm{~g}$ de fruto fresco), que fue estadísticamente igual a los frutos de las regiones Arequipa (Yunga y Suni) y Moquegua (Suni); mientras que la menor capacidad antioxidante se encontró en la muestra proveniente de Arequipa (Quechua) (Tabla 1). 
Tabla 1. Contenido de compuestos fenólicos, flavonoides y capacidad antioxidante del fruto de tumbo (Passiflora mollissima) de diferentes zonas agroecológicas

\begin{tabular}{|c|c|c|c|c|}
\hline $\begin{array}{l}\text { Procedencia } \\
\text { (Región) }\end{array}$ & $\begin{array}{l}\text { Fenoles totales } \\
\text { (mg EAG/100g) }\end{array}$ & $\begin{array}{l}\text { Flavonoides } \\
\text { (mg EQ/100g) }\end{array}$ & $\begin{array}{c}\text { DPPH } \\
\mathrm{IC}_{50}(\mathrm{mg} / \mathrm{mL})\end{array}$ & $\begin{array}{c}\text { FRAP } \\
\left.\text { (mmoles } \mathrm{Fe}^{2}+/ 100 \mathrm{~g}\right)\end{array}$ \\
\hline Arequipa (Yunga) & $507,25 \pm 99,52^{\mathrm{a}, \mathrm{b}}$ & $351,89 \pm 10,29 a, b$ & $0,41 \pm 0,01^{c}$ & $7,06 \pm 0,2^{a}$ \\
\hline Arequipa (Quechua) & $391,05 \pm 114,12^{b, c}$ & $223,54 \pm 119,18 c$ & $0,93 \pm 0,3^{b}$ & $5,04 \pm 1,39^{b}$ \\
\hline Arequipa (Suni) & $417,70 \pm 58,70^{a, b, c}$ & $429,99 \pm 36,74 a, b$ & $0,67 \pm 0,2^{b, c}$ & $7,05 \pm 0,06^{a}$ \\
\hline Cusco (Quechua) & $584,94 \pm 134,62^{a}$ & $445,62 \pm 7,94 a$ & $0,56 \pm 0,26^{b, c}$ & $8,38 \pm 0,22^{a}$ \\
\hline Cusco (Suni) & $287,22 \pm 2,58^{c}$ & & $1,45 \pm 0,1^{a}$ & \\
\hline Moquegua (Quechua) & $417,46 \pm 79,05^{a, b, c}$ & $314,72 \pm 63,43 b, c$ & $0,72 \pm 0,28^{b, c}$ & $6,54 \pm 1,24^{a, b}$ \\
\hline Moquegua (Suni) & $519,36 \pm 6,58^{a, b}$ & $403,87 \pm 3,51 a, b$ & $0,45 \pm 0^{c}$ & $7,48 \pm 0,2^{\mathrm{a}}$ \\
\hline
\end{tabular}

Los valores son los promedios $\pm \mathrm{DE}$ (desviación estándar).

Las letras diferentes dentro de la columna son significativas según la prueba de Tukey $(p<0,05)$.

En la tabla 2 se observa que el cerezo de la región Arequipa (Yunga) tuvo el mayor contenido de polifenoles $(181,81 \mathrm{mg}$ EAG/100 g), valor mayor que el fruto de la región Cusco (Quechua) (107,12 mg EAG/100 g), pero el fruto de la región Cusco (Suni) $(60,04$ mg EAG/100 g). El cerezo de la región Arequipa (Yunga) tiene el contenido más elevado de flavonoides (205,18 mg EQ/100 g), y el fruto de la región Arequipa (Quechua) muestra el valor más bajo (74,08 mg EQ/100 g).
La evaluación de la capacidad antioxidante, con la técnica DPPH, permite observar que el fruto del cerezo de la región Arequipa (Yunga) posee un valor IC50 de 2,1 mg/ $\mathrm{mL}$, que es el más bajo en comparación con los frutos de las otras regiones, lo cual significa que tuvo la mayor capacidad antioxidante. Asimismo, al evaluar la capacidad antioxidante con la técnica FRAP de los frutos originarios de Arequipa (Yunga), Arequipa (Quechua) y Cusco (Quechua), se observa que los valores encontrados no muestran diferencias significativas (Tabla 2).

Tabla 2. Contenido de compuestos fenólicos, flavonoides y capacidad antioxidante del fruto de cerezo (Prunus serotina) de diferentes zonas agroecológicas

\begin{tabular}{|c|c|c|c|c|}
\hline $\begin{array}{l}\text { Procedencia } \\
\text { (Región) }\end{array}$ & $\begin{array}{l}\text { Fenoles totales } \\
\text { (mg EAG/100g) }\end{array}$ & $\begin{array}{l}\text { Flavonoides } \\
\text { (mg EQ/ 100g) }\end{array}$ & $\begin{array}{c}\text { DPPH } \\
I_{50}(\mathrm{mg} / \mathrm{mL})\end{array}$ & $\begin{array}{c}\text { FRAP } \\
\left(\text { mmoles } \mathrm{Fe}^{2}+/ 100 \mathrm{~g}\right)\end{array}$ \\
\hline Arequipa (Yunga) & $181,81 \pm 34,1^{a}$ & $205,18 \pm 77,8^{a}$ & $2,1 \pm 0,01^{b}$ & $1,56 \pm 0,0^{a}$ \\
\hline Arequipa (Quechua) & $65,19 \pm 35,1^{b, c}$ & $74,08 \pm 5,5^{b}$ & $15,89 \pm 9,0^{a b}$ & $1,04 \pm 0,02^{\mathrm{a}}$ \\
\hline Cusco (Quechua) & $107,12 \pm 45^{a, b}$ & $96,97 \pm 2,2^{a, b}$ & $11,84 \pm 0,3^{a, b}$ & $1,59 \pm 0,2^{a}$ \\
\hline Cusco (Suni) & $60,04 \pm 14,1^{c}$ & & $18,34 \pm 0,14^{a}$ & \\
\hline
\end{tabular}

Los valores son los promedios \pm DE (desviación estándar).

Las letras diferentes dentro de la columna son significativas según la prueba de Tukey $(p<0,05)$.

En la tabla 3 se observa que las ratas de los grupos I y II que recibieron paracetamol $200 \mathrm{mg} / \mathrm{kg}$ de peso y tumbo liofilizado a las dosis de 200 y $400 \mathrm{mg} / \mathrm{kg}$ de peso, respectivamente, mostraron una menor concentración sérica de malondialdehído que el grupo control positivo, al que solamente se le administró paracetamol. Por el contrario, los grupos III y IV que recibieron la misma dosis de paracetamol (300 y $600 \mathrm{mg} / \mathrm{kg}$ del liofilizado de cerezo, respectivamente) mostraron valores séricos de malondialdehído mayores que los observados al inicio del tratamiento. 
Tabla 3. Niveles séricos de malondialdehído (MDA) en ratas tratadas con paracetamol y extractos liofilizados de tumbo (Passiflora mollissima) y cerezo (Prunus serotina)

\begin{tabular}{|lcc|}
\hline Grupos & $\begin{array}{c}\text { Antes } \\
\mathrm{nmol} \text { MDA/mL }\end{array}$ & $\begin{array}{c}\text { Después } \\
\mathrm{nmol} \text { MDA/mL }\end{array}$ \\
\hline Control negativo & $5,15 \mathrm{a}$ & $6,24 \mathrm{a}$ \\
\hline Control positivo & $6,3 \mathrm{~b}$ & $7,8 \mathrm{a}$ \\
\hline Experimental I & $8,44 \mathrm{a}, \mathrm{b}$ & $6,69 \mathrm{a}$ \\
\hline Experimental II & $10,05 \mathrm{a}$ & $6,24 \mathrm{a}$ \\
\hline Experimental III & $5,21 \mathrm{~b}$ & $7,36 \mathrm{a}$ \\
\hline Experimental IV & $6,04 \mathrm{a}, \mathrm{b}$ & $7,69 \mathrm{a}$ \\
\hline
\end{tabular}

Los valores son los promedios \pm DE (desviación estándar).

Las letras diferentes dentro de la columna son significativas, según t de Student $(p<0,05)$.

El estudio histopatológico del hígado de las ratas del grupo control negativo mostró una estructura normal bien conservada y sin signos de necrosis (figura 1A). Al grupo control positivo se le administró solamente paracetamol y mostró una estructura lobulillar conservada con focos de necrosis coagulativa de hepatocitos y hemorragia con infiltrado linfocitario portal leve (figura 1B). El hígado de la rata tratada con paracetamol y tumbo (400 mg/kg de peso corporal) muestra una estructura lobulillar normal, sin signos de necrosis, ni edema (Figura 1C); mientras que en el tejido hepático del animal tratado con paracetamol y cerezo (dosis de $600 \mathrm{mg} / \mathrm{kg}$ de peso) se observa edema leve de hepatocitos sin necrosis, estructura lobulillar conservada y congestión vascular leve focal (figura 1D).

(a)

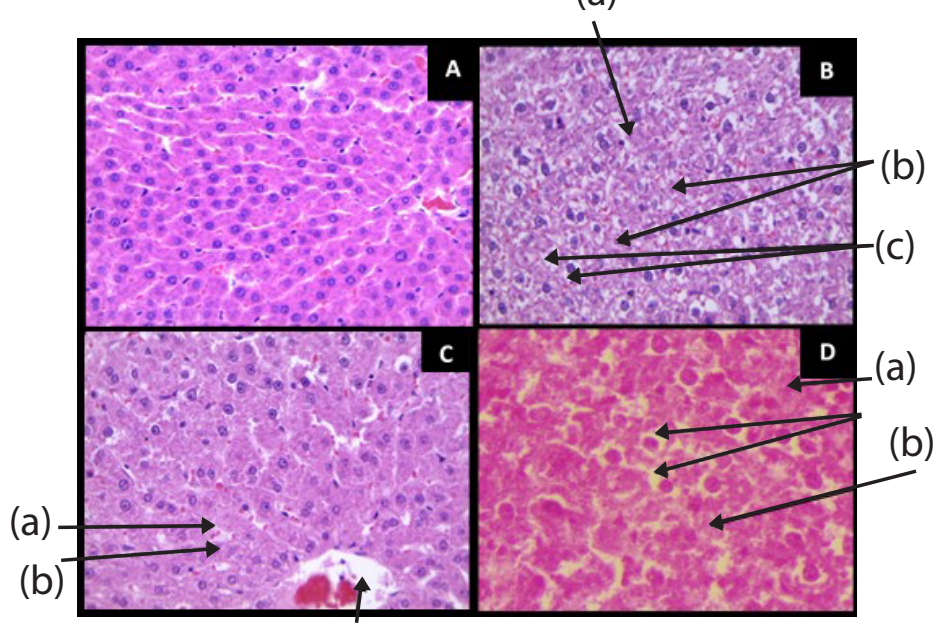

(d)

Figura 1. Hígado de rata luego del tratamiento

A. Control negativo, B. Control positivo con tratamiento de paracetamol $200 \mathrm{mg} / \mathrm{kg}$ de peso, C. Grupo experimental II con tratamiento tumbo $400 \mathrm{mg} / \mathrm{kg}$ peso y paracetamol $200 \mathrm{mg} / \mathrm{kg}$ de peso y D. Grupo experimental IV con tratamiento cerezo $600 \mathrm{mg} / \mathrm{kg}$ peso y paracetamol $200 \mathrm{mg} / \mathrm{kg}$.

(a) ausencia de núcleos (necrosis de hepatocitos, (b) citoplasmas claros y granulares, (c) edema intracelular de hepatocitos, (d) vena centro lobulillar. Coloración hematoxilina-eosina (40 X) 


\section{DISCUSIÓN}

El contenido de compuestos antioxidantes en un alimento depende de diversos factores como el genotipo, composición del suelo, clima, naturaleza del riego, altitud, etc. ${ }^{(16,24)}$. En nuestro estudio hemos evaluado los compuestos antioxidantes de dos frutos, el tumbo y el cerezo, que se consumen con frecuencia en Arequipa y también en regiones colindantes como Cusco y Moquegua. Los polifenoles son compuestos de conocidas propiedades antioxidantes que se encuentran como componentes de una gran diversidad de frutos ${ }^{(25)}$. El tumbo colectado en tres regiones del Perú (Arequipa, Cusco y Moquegua) tiene un elevado contenido de polifenoles (287,2 a 584,9 mg EAG/100 g), valores que son considerablemente mayores a la papaya de monte (167 mg EAG/100 g), al aguaymanto (154 mg EAG/100 g) y al tomate de árbol (130 mg EAG/100 g), mientras que las concentraciones de polifenoles del cerezo de la región Cusco (Suni) (60 mg EAG/100 g), de Arequipa (Quechua) (65 mg EAG/100 g) y la tuna roja (52 mg EAG/100 g) son menores. En cambio, el tumbo o curuba, como se le conoce en Colombia ${ }^{(13)}$, muestra un valor de $460 \mathrm{mg}$ EAG/100 g, que es menor que el del tumbo colectado en Arequipa (Yunga), Cusco (Quechua) y Moquegua (Suni). Sin embargo, otra muestra de curuba también colectada en Colombia ${ }^{(26)}$ tenía un contenido de fenoles totales de $635 \mathrm{mg}$ EAG/100 g, que es mayor que todas las muestras de tumbo colectadas en las diversas regiones del Perú. En un estudio que valora la capacidad antioxidante de frutas colectadas en Ecuador (27), la Passiflora mollissima muestra $1010 \mathrm{mg}$ EAG/100g de fenoles totales, cantidad que es superior a todos los frutos colectados con diferente ubicación geográfica.

El cerezo es un fruto con un volumen de antioxidantes que depende de la región donde se colecta. Así ocurre con el cerezo de Puebla (México), que contiene $325 \mathrm{mg}$ EAG/100 g de fenoles totales (13), cantidad que difiere notablemente de la concentración de otra planta de cerezo (746 mg EAG/100 g) que se recoge en el mismo ${ }^{(28)}$; ambos valores son mayores que los de las muestras colectadas en el Perú. El contenido de flavonoides ${ }^{(13)}$ de la primera muestra de cerezo (146 mg EQ/100 g) es menor al del cerezo de Arequipa (Yunga), aunque mayor que las muestras recogidas en Arequipa (Quechua) y Cusco (Quechua).

El contenido de flavonoides del cerezo colectado en Arequipa (Yunga) (205 mg EQ/100 g) fue más elevado que los valores mostrados por las diferentes variedades de frambuesa ${ }^{(29)}$, que son similares a los contenidos de flavonoides observados en el cerezo colectado en Arequipa (Quechua) y Cusco (Quechua). Mientras que los flavonoides del tumbo proveniente de las regiones estudiadas muestran valores mayores que las variedades de manzanas Rome beauty y Golden delicious ${ }^{(30)}$. Los elevados contenidos de polifenoles y flavonoides del tumbo hacen de este fruto una excelente fuente de antioxidantes, cuyo consumo proporcionaría protección frente a enfermedades crónicas no transmisibles.

La evaluación de la capacidad antioxidante con la técnica FRAP muestra que el cerezo tiene valores que se asemejan a frutas como fresa, limón, ciruela y naranja (31), cuyos valores están comprendidos entre 0,94 y 1,59 mmoles de $\mathrm{Fe}^{2}+/ 100 \mathrm{~g}$, pero son menores que la capacidad antioxidante del tumbo de todas las regiones estudiadas, cuyos valores están comprendidos entre 5,04 mmoles de $\mathrm{Fe}^{2+} / 100 \mathrm{~g}$, de la región Arequipa (Quechua) y 8,38 mmoles de $\mathrm{Fe}^{2+} / 100 \mathrm{~g}$ de la región Cusco (Quechua).

La evaluación de las propiedades bioactivas del fruto de Lycium barbarum ${ }^{(32)}$, con la técnica DPPH, muestra valores de la capacidad antioxidante que no difieren mayormente de unas muestras colectadas en China (IC50 : 0,795 mg/mL) y en Mongolia (IC50 : 0,784 mg/mL). En Grecia, algunos frutos recogidos tienen valores (IC50 entre 0,830 y 1,150 $\mathrm{mg} / \mathrm{mL}$ ) que dependen del mes en que fueron colectados. Estos valores son similares a los del tumbo de las regiones Arequipa, Cusco y Moquegua, pero son menores que los del cerezo originario de la región Arequipa (Quechua) y Cusco (Quechua y Suni).

En un estudio comparativo de las propiedades antioxidantes de varias frutas tropicales que utilizó la técnica DPPH (33) encontramos los siguientes valores IC50: guayaba $(2,11 \mathrm{mg} / \mathrm{mL})$, el plátano $(13,4 \mathrm{mg} / \mathrm{mL})$, la papaya $(3,5 \mathrm{mg} / \mathrm{mL})$ y la naranja $(5,4 \mathrm{mg} / \mathrm{mL})$, hallazgos más elevados que todas las variedades de tumbo colectadas en las regiones Cusco, Arequipa y Moquegua; es decir, que las frutas tropicales poseen menor capacidad antioxidante que el tumbo. Por el contrario, las capacidades antioxidantes de la guayaba, papaya y naranja son similares a las del cerezo de la región Arequipa (Yunga) (IC50: 2,1 mg/mL). Sin embargo, la capacidad antioxidante del plátano solo se compara con la del cerezo procedente de las regiones Arequipa (Quechua) y Cusco (Quechua y Suni).

La administración de $400 \mathrm{mg} / \mathrm{kg}$ de peso de paracetamol a las ratas durante siete días ocasionó un efecto hepatotóxico que fue evidente con el estudio histopatológico y por los valores de transaminasas, catalasa, superóxido dismutasa, etc. Estos efectos fueron revertidos por la administración de $500 \mathrm{mg} / \mathrm{kg}$ de peso de un extracto de Prunus serotina ${ }^{(34)}$, resultado similar al que hemos obtenido en condiciones análogas.

Las propiedades antioxidantes y hepatoprotectoras de los frutos de distintos orígenes muestran claramente que los polifenoles y flavonoides son compuestos bioactivos importantes que tienen un efecto protector en el hígado. En consecuencia, es necesario continuar con las investigaciones para establecer los mecanismos moleculares de la acción 
hepatoprotectora del tumbo y el cerezo.

Contribuciones de los autores: Juan Andrés Lopa Bolívar: Muestreo, obtención de resultados, análisis, interpretación de datos, redacción. María del Rosario Elsa Valderrama Valencia: Muestreo y análisis de datos. Nelva Consuelo León de los Santos: Asesoría administrativa, redacción y exposición en congreso del primer artículo y socialización de los resultados para el grupo meta. Luz Jani Lazo Jiménez: Obtención de resultados, análisis, interpretación de datos y redacción. Carlos Enrique Ballón Salcedo: Recolección de datos y obtención de resultados. Emilio Teodoro Guija Poma: Aspectos metodológicos y revisión crítica del manuscrito. Jean Pierre Llerena Madariaga: Recolección de la muestra y obtención de resultados.

Fuente de financiamiento: Este artículo fue financiado por la Universidad Nacional de San Agustín. UNSA-INVESTIGA. Contrato IBA-024-2016.Proyecto: Asociación de la variabilidad genética con las propiedades antioxidantes y nutracéuticas de prunus serótina var. salicifolia (cerezo), y passiflora mollissima (tumbo), para la revalorización de sus potencialidades.

Conflictos de interés: Los autores declaran no tener ningún conflicto de interés.

\section{REFERENCIAS BIBLIOGRÁFICAS}

1. Fehér J, Csomos G, Vereckei A. Free Radicals Reactions in Medicine. Springer-Verlag Berlin Heidelberg: Germany; 1987.

2. Hernández-García D, Wood CD, Castro-Obregón S, Covarrubias L. Reactive oxygen species: a radical role development? Free Radic Biol Med. 2010; 49(2): 130-43.

3. Halliwell B, Gutteridge JMC. Free radicals in Biology and Medicine. 4th ed. Oxford University Press: United Kingdom; 2007.

4. Cascales M. La toxicidad del oxígeno. Estrés oxidativo. En: Estrés oxidativo, envejecimiento y enfermedad. España; 1999. pp 9-46.

5. Virgili F, Marino M. Regulation of cellular signals from nutritional molecules: a specific role for phytochemicals, beyond antioxidant activity. Free Radic Biol Med. 2008; 45(9): 1205-16.

6. Pryor W. Oxidants and antioxidants. En: Natural antioxidants in human health and disease. Academic Press: USA; 1994.

7. Olatunji TL, Afolayan AJ. Comparative Quantitative Study on Phytochemical Contents and Antioxidant Activities of Capsicum annuum L and Capsicum frutescens L. Sci World J. 2019.

8. Li C, Feng J, Huang W-Y, An X-T. Composition of Polyphenols and Antioxidant Activity of Rabbiteye Blueberry (Vaccinium ashei) in Nanjing. J Agric Food Chem. 2013; 61(3): 523-31.

9. Biazotto KR, de Souza Mesquita LM, Neves BV, Cavalcante Braga AR, Pereira Tangerina MM, Vilegas W, et al. Brazilian Biodiversity Fruits: Discovering Bioactive Compounds from
Underexplored Sources. J Agric Food Chem. 2019; 67(7): 1860-76.

10. Cuzzocrea S, Riley DP, Caputi AP, Salvemini D. Antioxidant therapy: a new pharmacological approach in shock, inflammation and ischemia/ reperfusion injury. Pharmacol Rev. 2001; 53(1): 135-59.

11. Chen J, Jeppesen PB, Abudula R, Dyrskog SEU, Colombo M, Hermansen $K$. Stevioside does not cause increased basal insulin secretion or B-cell desensitization as does the sulphonylurea, glybenclamide: Studies in vitro. Life Sci. 2006; 78(15): 1748-53.

12. Wilson DW, Nash P, Buttar HS, Griffiths K, Singh R, De Meester F, et al. The Role of Food Antioxidants, Benefits of Functional Foods, and Influence of Feeding Habits on the Health of the Older Person: An Overview. Antioxidants (Basel). 2017; 6(4): 81.

13. Chaparro-Rojas DC, Maldonado ME, Franco-Londoño MC, UrangoMarchena LA. Características nutricionales y antioxidantes de la fruta curuba larga (Passiflora mollissima Bailey). Perspect Nutr Humana. 2014; 16(2): 203-12.

14. Guadalupe JJ, Gutiérrez B, Intriago-Baldeón DP, Arahana V, Tobar $\mathrm{J}$, Torres AF, et al. Genetic diversity and distribution patterns of Ecuadorian capuli (Prunus serotina). Biochem System Ecol. 2015; 60: 67-73.

15. Luna-Vázquez FJ, Ibarra-Alvarado C, Rojas-Molina A, Rojas-Molina JI, Yahia EM, Rivera-Pastrana DM, et al. Nutraceutical Value of Black Cherry Prunus serotina Ehrh. Fruits: Antioxidant and Antihypertensive Properties. Molecules. 2013; 18(12): 14597-612.

16. Correia S, Gonçalves B, Aires A, Silva A, Ferreira L, Carvalho R, et. al. Effect of Harvest Year and Altitude on Nutritional and Biometric Characteristics of Blueberry Cultivars. J Chem. 2016; (1): 1-12.

17. Carrión Jara AV, García Gómez CR. Preparación de extractos vegetales: Determinación de eficiencia de metódica [Tesis]. Ecuador: Universidad de Cuenca. Facultad de Ciencias Químicas; 2010.

18. Singleton VL, Rossi JA. Colorimetry of total phenolics with phosphomolybic-phophotungstic acid reagents. Amer J Enol Vit. 1965; 16: 144-58.

19. Zhishen J, Mengcheng T, Jianming W. The determination of flavonoid contents in mulberry and their scavenging effects on superoxide radicals. Food Chem. 1999; 64(4): 555-9.

20. Brand-Williams W, Cuvelier M, Berset C. Use of a Free Radical Method to Evaluate Antioxidant Activity. Food Sci Technol. 1995; 28(1): 25-30.

21. Benzie IF, Strain JJ. The Ferric Reducing Ability of Plasma (FRAP) as a Measure of «Antioxidant Power»: The FRAP Assay. Anal Biochem. 1996; 239(1): 70-6.

22. Ohkawa H, Ohishi N, Yagi K. Assay for lipid peroxides in animal tissues by thiobarbituric acid reaction. Anal Biochem. 1979; 95(2): 351-8.

23. Langhans, W. Food components in health promotion and disease prevention. J Agric Food Chem. 2017; 66(10): 2287-94.

24. Connor AM, Finn CE, Alspach P. Genotypic and environmental variation in antioxidant activity and total phenolic content among Blackberry and hybrid berry cultivars. J Am Soc Hortic Sci. 2005; 130(4): 527-33.

25. Wojdyło A, Nowicka P, Laskowski P, Oszmiański J. Evaluation of Sour Cherry (Prunus cerasus L.) Fruits for Their Polyphenol Content, Antioxidant Properties, and Nutritional Components. J Agric Food Chem. 2014; 62(51): 12332-45.

26. Contreras-Calderón J, Calderón-Jaimes L, Guerra-Hernández E, García-Villanova B. Antioxidant capacity, phenolic content and vitamin C in pulp, peel and seed from 24 exotic fruits from Colombia. Food Res Intern. 2011; 44(7): 2047-53.

27. Vasco C, Ruales J, Kamal-Eldin A. Total phenolic compounds and antioxidant capacities of major fruits from Ecuador. Food Chem. 2008; 111(4): 816-23.

28. Jimenez M, Castillo I, Azuara E, Beristain Cl. Antioxidant and antimicrobial activity of capulin (Prunus serotina subsp capuli) 
extracts. Rev Mex Ing Quim. 2011; 10(1): 29-37.

29. Liu M, Li XQ, Weber C, Lee CY, Brown J, Liu Rh. Antioxidant and antiproliferative activities of raspberries. J Agric Food Chem. 2002; 50(10): 2926-30.

30. Wolfe K, Wu X, Liu RH. Antioxidant activity of apple peels. J Agric Food Chem. 2003; 51(3): 609-14.

31. Szeto YT, Tomlinson B, Benzie IFF. Total antioxidant and ascorbic acid content of fresh fruits and vegetables: implications for dietary and planning and food preservation. British J Nut. 2002; 87(1): 55-9.

32. Kosar M, Altintas A, Kirimer N, Baser KHC. Determination of the free radical scavenging activity of Lycium extracts. Chem Nat Compd. 2003; 39(6): 531-5.

33. Lim, YK, Lim TT, Tee JJ. Antioxidant properties of several tropical fruits: A comparative study. Food Chem. 2007; 103(1): 1003-8.

34. Zhou H-C, Wang H, Shi K, Li J-M, Zong Y, Du R. Hepatoprotective Effect of Baicalein Against Acetaminophen-Induced Acute Liver Injury in Mice. Molecules. 2018; 24(1): 131.

\section{Correspondencia:}

Juan Andrés Lopa Bolívar

Dirección: Urb. Paisajista Chilina B-1A Yanahuara. Arequipa, Perú.

Teléfono: +51958534000

Correo electrónico: jlopa@unsa.edu.pe

Recibido: 22 de febrero de 2021

Evaluado: 03 de marzo de 2021

Aprobado: 05 de abril de 2021

\footnotetext{
(c) La revista. Publicado por Universidad de San Martín de Porres, Perú. (cc) Br Licencia de Creative Commons Artículo en acceso abierto bajo términos de Licencia Creative Commons Atribución 4.0 Internacional. (http://creativecommons.org/licenses/by/4.0/)

ORCID iDs

Juan Lopa

María Valderrama

Nelva León

Luz Lazo

Jean Pierre Llerena

Carlos Ballón

Emilio Guija-Poma

(1) https: / / orcid.org/0000-0003-1413-7537
https: / / orcid.org/0000-0002-1515-3195
https: / / orcid.org/0000-0001-7458-4609
https: / / orcid.org/0000-0001-6773-8550
https: / / orcid.org/0000-0003-2562-367X
https: / / orcid.org/0000-0002-9794-0968
(1) https: / / orcid.org/0000-0002-2522-2772
} 